\title{
MRI Images Techniques
}

\author{
Ms. Pallavi L. Patil ${ }^{1}$, Mr. V.B.Raskar ${ }^{2}$ \\ $\operatorname{ME}\left(2^{\text {nd }}\right.$ Year)Student, Department of Electronics and Telecommunication Engineering \\ (Signal processing) JSPM Wagholi, Pune, Maharashtra, India ${ }^{1}$ \\ Assistant professor, Department of Electronics and Telecommunication Engineering \\ (Signal processing) JSPM Wagholi, Pune, Maharashtra, India ${ }^{2}$
}

\begin{abstract}
Magnetic Resonance Imaging (MRI) is beneficial and effective diagnostic tool in basic analysis, clinical investigation, and unwellness identification since it provides each chemical and physiological info regarding the tissue underneath investigation. however once noise get introduced in mister pictures, it decreases the image quality ,Image analysis and becomes tough to diagnose it accurately. A trade off between noise reduction and preservation of actual image options must build in such manner that enhance the diagnostically relevant image content. so noise reduction remains difficult task. This paper proposes wave domain denoising for removal of Rician noise from MR Image. This methodology heavily depends on alternative of threshold parameter that successively determines the potency of denoising.
\end{abstract}

Keywords: Magnetic resonance imaging, Rician noise, Threshold, denoising

\section{INTRODUCTION}

MRI is imaging technique used in radiology for qualitative and quantitative analysis it shows that this visualizing the internal structure of tissues and organs in algorithm is more efficient than the wavelet methods in the body. It is used for assessing the pathological or image denoising particularly for removal of Gaussian physiological alternations of living tissues .It differs from noise. This method can obtain high PSNR than wavelet other imaging modalities such as X-ray ,Computed based denoising algorithm using MR images in the Tomography in such manner that it can characterise and presence of AWGN[10] Rohini Mahajan proposed wavelet discriminate among tissues using their biochemical and domain denoising for the removal of Rican noise from MR physical properties.Also, without moving patient it can images and this method depends on the selection of produce sectional image of equivalent resolution. This threshold value which in turn predicts the efficiency of adds to its versatility and diagnostic utility which gives it denoising method. Symlet filter (sym6) is used for special advantage for surgical treatment planning. One denoising purpose which tends to preserve the shape of more advantage is that it does not cause the harmful reflectance peak and smoothness value of data point[16]. radiations to the patient [19].For clinical diagnosis, the S.Jakhade introduced an algorithm that uses wavelet based visual quality of magnetic resonance images plays an multi resolution analysis and adaptive filtering which can important role. But during acquisition or transmission efficiently remove noise from image. By doing these images are corrupted with noise, which hinders the comparative study it has been proved MRI with Rican and medical diagnosis based on these images. Thus, denoising speckle type of noise, adaptive bilateral filter is best of image is necessary and foremost step to be taken suitable for MRI denoising in wavelet domain.[18] For beforethe image data to be analysed .Image denoising is image denoising ,Kanwaljot singh et al worked on still challenging as removal of noise causes artifacts and Haarand Daubechies transform. Initially ,image is image blurring. Different algorithms are used for decomposed using Haar and Daubechies transform then denoising which depends on noise model and modelling of the level of hard and soft threshold is selected and then by noise depends on many factors like data capturing calculating and comparing the PSNR of an image for instrument ,transmission media ,quantization of image every wavelet, desired wavelet is assigned which gives .This paper explains denoising of MRI images using more PSNR of respective image. From this comparison it thresholding techniques through wavelet.

A brief survey of representative techniques denoising of is found that $\mathrm{dB} 3$ wavelet is more efficient which enhance the visual quality of images and thus it helps in effective diagnosis.[24] Neelabh Sukatme used UDWT for adaptive thresholding technique based on wavelet diagnosis of MRI which is based on idea of no decimation. coefficients along with neighbourhood pixels filtering It omits the down sampling at the It gives more precise algorithm [NPFA] for noise suppression of MRI. This information .Comparing with DWT on the basis of PSNR stastical model describes a new method for denoising by values it has been proved that UDWT found to be good in fusing the wavelet denoising technique with optimized terms of quality metrics.[14] Rupindrapal kaur et al threshholding function This technique has given presents a wavelet based technique for detection and significantly superior image quality by preserving edges, removal of noise in MRI images. Though the set of producing better PSNR value[6]. Image denoising using wavelet has some common properties but each has certain contourlet transform is proposed by S.Satheesh. Based on unique properties of image decomposition denoising and 
reconstruction. Result obtained by quantitative and qualitative comparison of daubechies wavelet and maltt wavelet transform for Gaussian noise shows that image denoising using fast wavelet transform has better results compared to daubechies wavelet with less proceesing time.Also it reduces memory requirement ,complexity and increases the flexibility, Also it enhances the visual quality of MRI by achieving high PSNR and low MSE[1]. Shashikant Agrawal et al proposed a medical image denoising algorithm using DWT .They have presented the generation of DWT methods for 2-D case. From the result it shows that the bio orthogonal wavelet (bior1.3) gives best result compared to other and also optimal thresholding gives better denoised result among the three thresholding techniques.[2] A new approach introduced by zuvria to enhance denoising of MRI that exploits the sparseness and self similarity properties of images. This is based on three dimensional moving window discrete cosine transform hard thresholding and rotationally invariant version of non-local means filter .This proposed method run in reasonable time and can be used for most research and clinical settings. [3] Paul Bao proposed MRI image denoising using an adaptive wavelet thresholding. This scheme multiplies the adjacent wave subbands to amplify the significant feature and then applies thresholding to the mulitscale scheme which gives high SNR and also preserves edges of the image. [4]

\section{II . NOISES IN MRI}

During acquisition or transmission MRI images are largely corrupted by noise. Also, noise is also made as a result of imperfect instrument used during processing, interference and compression [7]. Image noise can be defined as random variation of brightness or color information image produced by the sensor and circuitry of the scanner. Noise in MRI poses a lot of problem to medical personnel by interfering with interpretation of MRI for diagnosis and treatment of human .Image noise in large measures contributes high hazards faced by human [8].In the digital images like MRI, noise are low as well as high frequency components. Removing high frequency components is very easy as comparatively with low frequency components as real signal and low frequency noise can not be distinguished easily [7]. Noise in MRI mostly obeys Rician distribution. The term rician noise is used to the error between underlying image intensities and the observed data. As it has non zero mean, its mean depends on the local intensity in the image. Also, rician noise is signal dependent and particularly problematic in high resolution, low signal to noise ratio regime where it not only causes random fluctuations but also introduces as signal dependent bias to the data that reduces image contrast. As bias field signal is low frequency signal which corrupts MRI images because inhomogenities in the magnetic field of MRI machines. It blurs the images and reduces the high frequency content of image such as edge, contours and also alters the intensity values of image pixels. Because of this tissues have different grey level distribution across image. Image processing algorithm like segmentation, classification or texture analysis use the grey level values of that image pixels which will not give satisfactory result. The preprocessing is required for correction of bias field signal before submitting corrupted MRI to such algorithm.[16] Rician noise affects the image in both quantitative and qualitative manner and thus it hinders image analysis, interpretation and feature detection [16].So denoising method is required which removes this noise. Denoising is nothing but the removing noise from image while retaining the original quality of the image. The great challenge of image denoising is how to preserve the edges and all fine details of an image while suppression of noise. It still remains challenge for researchers as noise removal introduces artifacts and causes blurring of the images [1]. So, it is necessary to develope an efficient denoising technique to avoid such knowledge corruption.

\section{METHODOLOGY}

Denoising of MRI images, initially take MRI image which should be in the form of jpg, tif or png. Add noise like (pepper and salt noise, rician noise etc) in the original image to obtain noisy MRI image. Using the wavelet transform, we decompose the image to lower depth. Wavelet decomposition is done using Discrete Wavelet Transform (DWT). DWT is used to decompose image to $n$ no. of levels having four sub bands at each level(LL, LH, HL, HH sub bands), from which we get wavelet coefficients for further processing. Calculate the noise variance for each sub band. Apply thresholding technique to this components according to shrinkage rule and its types. By doing this ,noisy components can be easily removed from MRI image. Then, invert the discrete wavelet transform(IDWT), to obtain denoised image. Efficiency can be checked by comparing the parameters like signal to noise ratio (SNR), Peak signal to noise ratio (PSNR), Mean square error (MSE) of the noisy and denoised images.

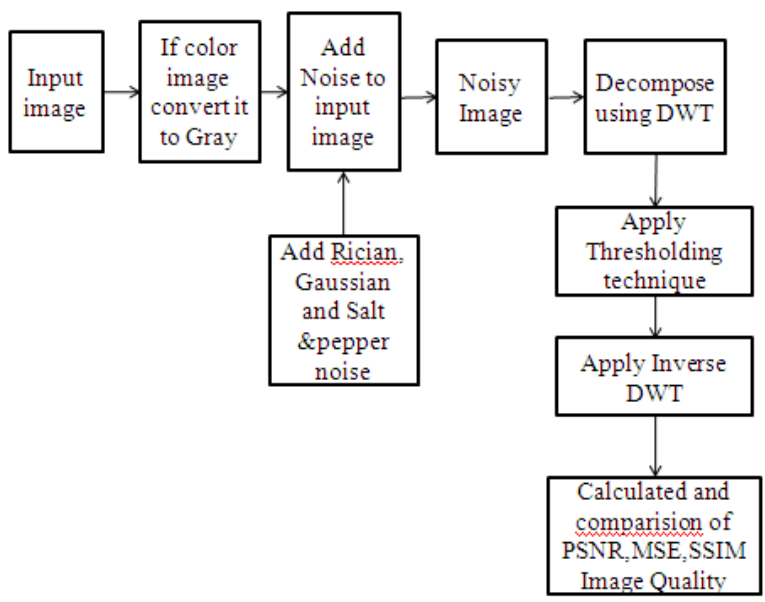

Fig. 1 Block diagram

\section{WAVELET DENOISING}

Wavelet denoising attempts to remove noise which is present in the signal while retaining all the signal characteristics regardless of its frequency contents [16].In practise, the wavelet transform implemented with 
reconstruction bank using wavelet families. The use of wavelet transform as filter bank called discrete wavelet transform (DWT).The DWT provides better spatial and spectral localization of signal formation.[12].Also it provides non-redundant and unique representation of signal .Due to this ,signal and noise are easily separated in wavelet domain. Here, the signal is decomposed into subsignals corresponding to different frequency content. The result of DWT is a multilevel decomposition which will generate wavelet coefficients. These coefficients include various approximation and detail coefficients at various levels and it leads to four different sub-bands as $\mathrm{HH}, \mathrm{HL}, \mathrm{LH}$ and LL at each level. The approximation subband consists of the so called scaling coefficients (LPF) whereas the detail sub-band (HPF) are composed of wavelet coefficients. The role of decomposition within the analysis of $\mathrm{N}^{*} \mathrm{~N}$ image can be explained as:

-Initial LPF of the rows blurs the image values on each row followed by low pass filtering.

- on the columns that finish in associate occasional pass approximation of the full image.

- LPF of the rows followed by HPF of the columns highlights the changes that occur between the rows horizontal details

-Initial HPF of the initial rows of the image highlights the changes between elements in any resultant LPF of the columns blurs the changes which can occur between the rows therefore providing the vertical details

- HPF of the rows followed by high pass filtering of the columns entirely changes that unit of measurement neither horizontal unit of measurement emphasized. This sequence provides the diagonal details of image. [23]

\begin{tabular}{|c|c|c|}
\hline LL2 & HL2 & \\
LH2 & HH2 & HL1 \\
& \\
LH1 & HH1 \\
\hline
\end{tabular}

Fig 2 .Two level 2-D DWT of image

The digital transformation of image for computer processing requires digitization, so here we are using 2-D discrete wavelet transform which decompose image several sub-bands. In case of two dimensional image ,after DWT the image is divided into four corners, upper left of the original, lower left of corner of vertical details upper right of the horizontal details and lower right corner of the image detail.[23].

\section{WAVELET THRESHOLDING}

Threshoding is a non-linear technique, operates on one wavelet coefficients at a time.The term wavelet coefficients is explained as decomposition of image data into wavelet coefficients, comparing the detail coefficients

with threshold value and shrinking there coefficients close to zero to take away the effects of noise. Thresholding distinguish between the coefficients because of noise and so the one consisting of important signal details. The choice is degree important aspect as its plays major role in removal of noise in image. Only care should take for preserving the edges of denoised image. [16]For this Donoho and Johnstone proposed a non-linear strategy for thresholding. In their approach, thresholding can be applied by using either hard or soft thresholding method which is also called as shrinkage rule.[12]

\section{A. Hard Thresholding}

In this approach, wavelet coefficients below threshold value are set to zero and keeps the other unchanged it does not affect the detail coefficients that are greater the threshold value.

$\overline{C s}(k)=\operatorname{sign} C(k)|C(k)|$

$$
0
$$

$$
\begin{aligned}
& \text { if }|c(K)|>\lambda \\
& \text { if }|C(k)| \leq \lambda
\end{aligned}
$$

Where $\lambda$ is threshold

\section{B. Soft Thresholding}

In this approach, coefficients underneath the edge square measure set to zero however scales those that square measure left. It can be define as

$$
\begin{array}{cc}
\overline{\mathrm{Cs}}(\mathrm{k})=\operatorname{sign} \mathrm{C}(\mathrm{k})|\mathrm{C}(\mathrm{k})|-\lambda & \text { if }|\mathrm{c}(\mathrm{K})|>\lambda \\
0 & \text { if }|\mathrm{C}(\mathrm{k})| \leq \lambda
\end{array}
$$

The important point thresholding method is to find the appropriate value for threshold. The threshold value is estimation of noise level .It is generally calculated from the standard deviation of the detail coefficients. Donho proposed a good estimator for the wavelet denoising, which is given as

$$
\sigma=\frac{\text { median }\left(\mathrm{d}_{\mathrm{L}-1, \mathrm{k}}\right)}{0.6745} \quad \mathrm{k}=0,1, \ldots \ldots, 2^{\mathrm{L}-1}-1
$$

Where $\mathrm{L}$ is number of decomposition levels. This median selection made on the detail coefficients of signal to analysed. [12]

\section{Global Threshold}

This is one of the threshold selection algorithms which has a universal threshold given by[2] .

$$
\lambda=\sigma \sqrt{2 \log (\mathrm{N})}
$$

Where $\mathrm{N}$ is that size of coefficients arrays and $\sigma \mathrm{i} \mathrm{s}$ the noise variance of the signal.

\section{Mean Square Error (MSE)}

This is one of the error criteria used in this work.MSE is the squared difference between the original and the denoised image. This gives us the difference between original image and the denoised image. Its given by:

$$
\operatorname{MSE}=\frac{1}{M N} \sum_{m=0}^{M-1} \sum_{n=0}^{N-1}\left(y(m, n)-y^{\prime}(m, n)\right)^{2}
$$


between Where $y(m, n)$ is the original image and $y^{\prime}(m, n)$ is denoised image with relation to image dimension $(\mathrm{m}, \mathrm{n}) \cdot[8]$

\section{E. Signal to Noise Ratio (SNR)}

SNR is very useful way of comparing the relative amount of signal and noise .signal to noise ratio estimates the quality of a reconstructed image compared with an original image.High measures will have very little amount of noise and opposite is true for low ratios.[16]

$$
\operatorname{SNR}=\frac{\sigma_{\mathrm{s}}}{\sigma_{\mathrm{n}}}
$$

Where $\sigma_{\mathrm{s}}$ and $\sigma_{\mathrm{n}}$ are the variance of the original image and recorded image. This gives us the difference between original image and the denoised image.

\section{F. Peak Signal to Noise Ratio (PSNR)}

It is the ratio between maximum possible power of a signal and the power of corrupting noise that affects the quality and reliability of its representation. PSNR is calculated as

$$
\text { PSNR }=10 \log _{10}\left(\frac{\mathrm{MAX}^{2}}{\mathrm{MSE}}\right)
$$

Where MSE is mean square error and MAX is the maximum pixel value of image [8].

\section{RESULT}

We used MATLAB to implement the de-noising algorithm. MATLAB has a wavelet toolbox and functions which are very convenient to do the DWT. A usual way to de-noise is to find a processed image such that it minimizes mean square error MSE and increases the value of the PSNR.

Keeping MRI images same we have varied noise added to image, wavelet family and threshold type (hard \& soft Thresholding). PSNR for rician noise is better.So, We used MATLAB to implement the de-noised algorithm. MATLAB has a wavelet toolbox and function which are very convenient to do the DWT.

In below Fig 3. is shows 2D discreat wavelet transformer

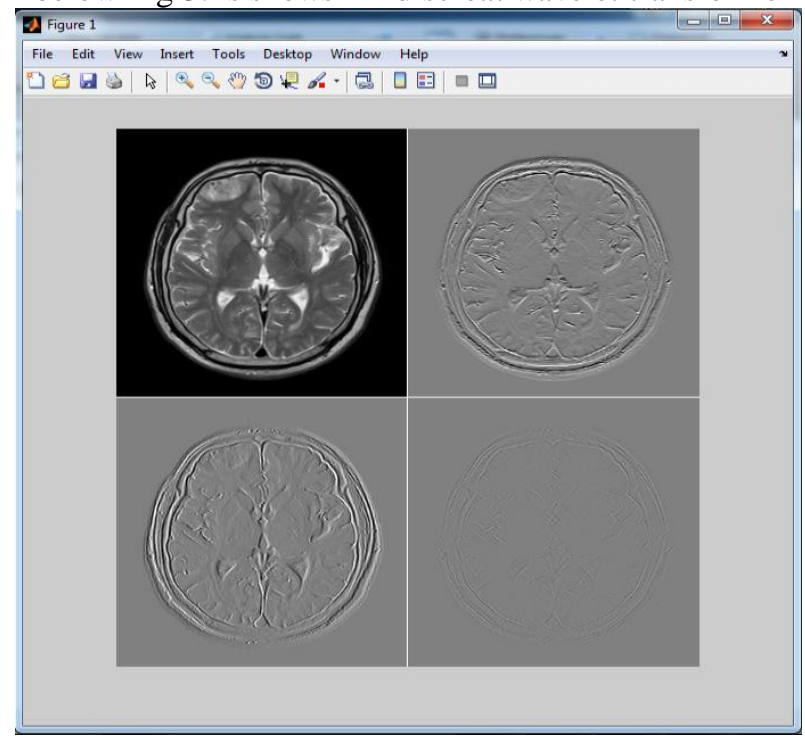

Fig 3. 2D discrete wavelet transforms
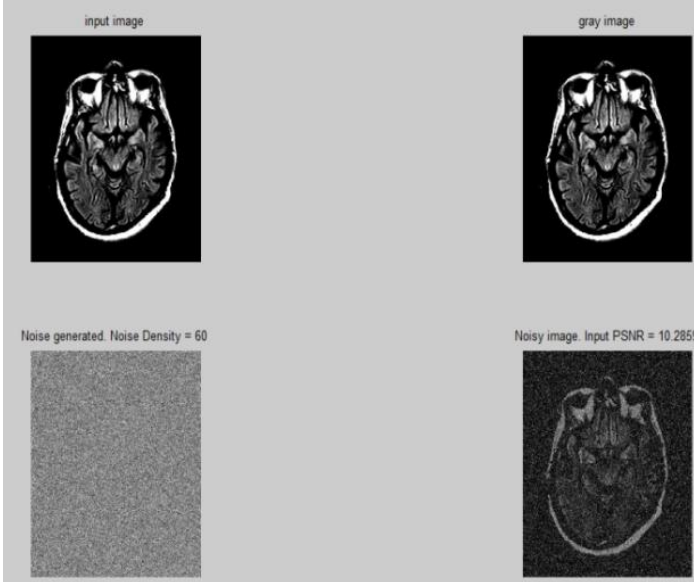

In Fig 4. Calculation of Input PSNR

In above Fig Calculation of PSNR for different wavelet families. For denoising of MRI images, take MRI image which should be in the form of jpg, tif or png which is then converted to gray image if it is RGB image for further processing. Next is add noise like rician noise in the original image to obtain noisy MRI image to check the efficiency of this method by comparing parameters like Input Peak signal to noise ratio.

\section{CONCLUSION}

In this paper, we have presented the denoising method based on wavelet thresholding which offers high quality and flexibility for noise problem of signal and images. By comparing soft and hard thresholding, it is seen that PSNR values for soft is better as compared to hard thresholding when it is used for rician noise and also MSE is less in case of soft thresholding. Wavelet denoising technique works well for rician type of noise as compared to Gaussian and salt \& pepper noise.

\section{REFERENCES}

[1] Rupinderpal Kaur ,Rajneet Kaur "Image Denoising supported wavelet Techniques exploitation Threshold for for Medical photos "International Journal of computer Trends and Technology (IJCTT), voiume four issue eight aug2013

[2] Shashiant Agrawal yogesh bahendwar " Denoising of imaging photos exploitation Thresholding Technique Through Wavelet'Intrnational Journal of computer Applications in Engineering Sciences, voll,issue third,2011

[3] Mrs.N.mymoon Zuviria,MRs.V.kuppammal "Sparseness and selfsimilarity supported Rician noise removal of resonance image" Tnternational Journal of Advanced analysis in computing and package Engineering,Voi.3,issue 3,March 2013

[4] Paul Bao And Lei Zhang"Noise reduction for resonance image via adaptative multiscale product Thresholding"IEEE transction of medical imaging,vol 22,No.9,sept 2013

[5] S. KALAVATHY" application of subband thresholding technique with neighbourhood constituent filtering for denoising imaging images" International of field and Technology (IJEST) ISSN : 0975-5462 Vol. 4,Issue2,February2012

[6] Olawuyi, N.J." Comparative Analysis Of WaveletBased Denoising algorithmic programs On organ resonance Images" Afr J Comp \& ICT Olawuyi et al - Comparative Analysis of Wavelet-Based Denoising algorithmic rule Vol four. No. 1. June 2011

[7] Burhan Ergen" Signal and Image Denoising exploitation riffle Transform"www.intechopen.com [10] S.Satheesh" medical image denoising exploitation adaptative threshold supported Advanced 
Computing contourlet transform": a worldwide Journal ( ACIJ ), Vol.2, No.2, March 2011

[8] insufficient Garg" up Segmentation by Denoising Brain imaging photos through Interpolation Median Filter in ADTVFCM" International Journal of computer Trends and Technologyvolume4Issue2- 2013

[9] Burhan Ergen" Signal and Image Denoising exploitation wavelet Transform

[10] S.Satheesh" medical image denoising exploitation adaptative threshold primarily based onAdvanced Computing contourlet transform" : a worldwide Journal ( ACIJ ), Vol.2, No.2, March 2011

[11] Neelabh Sukhatme" Denoising of imaging footage use Domain transform primarily based methods"

[12] Partha Sarathi Mukherjee" economical Bias Correction For imaging Image Denoising" college of Statistics, University of polestar State

[13] Rohini Mahajan, Akshay Girdhar" riffle primarily based denoising of imaging images" International Journal ofComputer Science analysis and Application 2010, Vol. 01, Issue. 01
[14] R.Gayathri, "A Survey on Image Denoising Algorithms (IDA) "International Journal of Advanced analysis in Electrical, physics and Instrumentation Engineering Vol. 1,Issue five,November2012

[15] Swapnil Jakh Kedar Phatak Aniket Nene drink," riffle Domain Filtering of adult male Image Sequences with applicable Filtering Approach supported adult male Image Type"2012

[16] Hossein Rabbani, Wavelet-Domain Medical Image Denoising victimisation quantity Laplacian Mixture Model" ieee transactions on medical specialty engineering, vol. 56, no. 12, Dec 2009

[17] Bhupal SinghClassification of Brain imaging in riffle DomainInternational Journal of physical science and computing Engineering 879Available on-line WWW.ijecse.orgIJECSE,Volume1,Number 3

[18] S.Satheesh" medical image denoising victimisation reconciling threshold based mostly onAdvanced Computing contourlettransform" : a global Journal ACIJ)Vol.2,No.2,March2011 